\title{
Risks of Simultaneous Coinfection of Dengue and COVID-19 in Bangladesh: Challenges and Recommendations
}

\author{
Md. Sahidur Rahman ${ }^{1, *},(\mathbb{D})$, Tazrina Rahman ${ }^{1,2}$ \\ ${ }^{1}$ Department of Research and Innovation, One Health Center for Research and Action, D/137, Bishaw Bank R/A, Akbarshah, Chattogram 4207, Bangladesh \\ ${ }^{2}$ Department of Microbiology and Virology, Chattogram Medical College Hospital, Chakbazar, Chattogram 4203, Bangladesh
}

\author{
ARTICLE INFO \\ Article History \\ Received 23 September 2020 \\ Accepted 12 November 2020 \\ Keywords \\ Challenges \\ coepidemic \\ coinfection \\ COVID-19 \\ dengue fever \\ healthcare facility
}

\begin{abstract}
The current global Coronavirus Disease 2019 (COVID-19) pandemic is compounding on populations susceptible to tropical illnesses such as dengue in different developing countries such as Bangladesh. The growing concern is that Bangladesh is a dengue-endemic zone and the peak transmission occurs in the monsoon season (June to October). In the most recent monsoon, a total of 354 dengue cases have been confirmed until July 27, 2020, data-driven from only 41 hospitals alone. A 53-yearold male patient was found to be coinfected with COVID-19 and dengue fever in Bangladesh. Concerns arise as hospitals are increasingly denying admission to patients. Moreover, reports of false-positive results in dengue screening tests recorded in different countries further exacerbate the issue. These conditions could postpone the early diagnosis of COVID-19 cases and aggravate the situation. In addition, the overwhelming wave of dengue cases would be a challenge for the vulnerable healthcare system of the country, which is already under strain because of the COVID-19 pandemic. Failure to establish and implement proper policies might lead to dengue outbreak with the burdens of the concurrent COVID pandemic, resulting in the collapse of the health and social systems, as well as the economic growth of the country.
\end{abstract}

(C) 2020 Dr. Sulaiman Al Habib Medical Group. Publishing services by Atlantis Press International B.V. This is an open access article distributed under the CC BY-NC 4.0 license (http://creativecommons.org/licenses/by-nc/4.0/).

\section{INTRODUCTION}

Similar to the rest of the world, Bangladesh has also been struggling to tackle the spread of Coronavirus Disease 2019 (COVID-19), which is caused by a novel coronavirus, severe acute respiratory syndrome coronavirus 2 . Since the declaration of the first three COVID-19 confirmed cases on March 8, 2020, the COVID-19 curve has been increasing in Bangladesh. To date (September 7, 2020), a total of 325,157 confirmed cases with 4479 deaths have been recorded [1]. Amidst this COVID-19 crisis, the monsoon has already arrived as Bangladesh has a subtropical climate with a rainy monsoon season. The heavy rains in this period cause a sudden spike in the Aedes mosquito population, which is the vector of dengue fever. In 2019, the country witnessed the worst dengue fever outbreak, with 101,354 infection cases and more than 276 deaths since the time of the first major outbreak in 2000, according to the data made available by the Directorate General of Health Services [2].

As precautionary measures for the COVID-19 pandemic, people are recommended to stay indoors by healthcare professionals, and a countrywide lockdown is being attempted. This condition creates a higher risk of dengue infection in residential areas as Aedes mosquitoes are capable of breeding in households and feed mostly during the daytime. The Dhaka North City Corporation found

"Corresponding author. Email: sahid.dvm@gmail.com

Peer review under responsibility of the Dr. Sulaiman Al Habib Medical Group that more than $60 \%$ of houses in Dhaka city were potential Aedes breeding grounds, and the "National Malaria Elimination and ATD Control Programme" found larvae in $13.62 \%$ of the houses [3]. Up until July 2020, a total of 354 cases of dengue have been reported. The number of unreported dengue patients is expected to be high as the government collects patients' information from only 41 hospitals out of more than 3000 [3]. This indicates an imminent crisis involving an overwhelming wave of dengue patients who will emerge during or after this monsoon season. Other Southeast Asian countries are also grappling with a rise in dengue cases. The National Environment Agency of Singapore has reported 7502 dengue fever cases until May 15, 2020, which was almost twice the number of cases found during the same period in 2019. India, Pakistan, Indonesia, Thailand, and Malaysia also confirmed the rapid increase of dengue patients in different susceptible zones of their respective territories [4].

\section{AN INSIGHT INTO THE CHALLENGES}

Recent studies have provided evidence that dengue and COVID-19 have shared clinical and laboratory features that cause difficulty in early diagnosis and distinguishment among the patients $[5,6]$. The onset of both diseases is manifested with fever. In the case of dengue, it causes a sudden development of high fever. By contrast, COVID-19 cases show mild fever with respiratory illnesses such as cough or shortness of breath. Respiratory distress is also found in severe dengue cases. Nausea, vomiting, abdominal pain, and 
headache are some of the overlapping symptoms in both illnesses [7]. A 53-year-old male patient with fever, cough, muscle pain, and breathing complications was the first person to be recognized as positive for both coronavirus and dengue fever in Bangladesh [8]. One classical sign of dengue is skin rashes (petechiae), but a COVID-19 patient in Thailand who had skin rashes early on was misdiagnosed as having dengue [9]. A patient living in Reunion Island, a French overseas department located in the Indian Ocean, was confirmed as a case of dengue and COVID-19 co-infection. The patient had rashes, arthromyalgia, and dyspnea with polypnea, which are common physical signs and diagnostic criteria similar in both diseases [10]. Another study in Pakistan found positive results for both dengue serological test and COVID-19 reverse transcriptase polymerase chain reaction (RT-PCR) test in at least $25 \%$ of patients (five out of 20 ) with $60 \%$ mortality rate in coinfected patients. This revealed the lower number of white blood cells, neutrophils, lymphocytes, and platelets in blood of coinfected patients, who eventually died at hospital [11]. In Indonesia, a patient who passed away was found to be both dengue serological test positive and RT-PCR positive for COVID-19 [12]. Both diseases lead to multiorgan involvement and shock at the later stage. Furthermore, false positive results in dengue screening tests could hamper the diagnosis of COVID-19 infection and facilitate further spread of the virus [13]. Pre-existing dengue-antibodies also have the potential to affect the results of COVID-19 serology tests through cross-reactivity [14]. Moreover, owing to the COVID-19 pandemic, many hospitals are unable to provide regular healthcare services. Many patients have even been denied admission without a COVID-19 test report, and the government health department has also postponed different routine health programs such as surveillance and vaccination. These interruptions in the healthcare system lead to the delay and/or misdiagnosis as well as hindrances in the treatment of dengue. Residents might also be unaware of the progress of another disease until the complications become serious, as they are in stress and fear of the ongoing COVID-19 infection. As a result, the epidemic of dengue this year might present huge challenges to the already stressed healthcare facilities of the country.

\section{RECOMMENDATIONS}

It is high time for the implementation of effective strategies to contain the growing public health threats caused by the dengue epidemic and the COVID-19 pandemic. Healthcare workers should undertake precautionary measures for themselves and be made aware of the complexity created by dengue fever and COVID-19. There must be strict infection prevention and control measures from primary to tertiary healthcare levels to ensure sufficient clinical care to dengue cases in this pandemic situation. The circulation of proper guidelines from the regulatory body to primary healthcare units might assist in the early differentiation of both cases as well as reduce severe consequences. Any warning signs such as mucosal bleeding, abdominal pain, vomiting, fluid accumulation, and sluggish activities should be given the highest priority in terms of management. In each of the affected localities, efforts must be taken for differential diagnosis in laboratory diagnostic set up as the clinicoepidemiological features are not sufficient to differentiate the coinfection of COVID-19 and dengue fever [11]. Required medicines, equipment, and testing facilities should also be provided to marginal healthcare units for strengthening the healthcare system to prepare for the overwhelming cases of dengue fever within the ongoing burden of COVID-19 patients. Immediate development of dengue monitoring cells through the active participation of health departments and city authorities for case management and vector control programs, especially in big cities, is crucial. Household mosquito breeding sources should also be eliminated considering that the public is requested to stay indoors for a prolonged period. Encouraging community members to eliminate or control mosquito breeding sites, in and around their properties, is a most feasible strategy [14]. The regular delivery of health alerts using social media, in combination with traditional media, are needed to prevent the spread of rumors and encourage early reporting of new cases. Future directions could include comprehensive research that explores the potential of coinfection between coronavirus and arbovirus.

\section{CONFLICTS OF INTEREST}

The authors declare they have no conflicts of interest.

\section{FUNDING}

This research did not receive any specific grant from funding agencies in the public, commercial, or not-for-profit sectors.

\section{REFERENCES}

[1] IEDCR. Institute of epidemiology disease control and research. 2020. Available from: https://www.iedcr.gov.bd/ (accessed September 2, 2020).

[2] DGHS. Daily Dengue Status Report. 2020. Available from: https:// dghs.gov.bd/index.php/en/mis-docs/epi/81-english-root/5200daily-dengue-status-report (accessed September 2, 2020).

[3] Newage Bangladesh. Spread of dengue feared as many patients go unreported 2020 (accessed September 5, 2020).

[4] Dengue virus net. Dengue Breaking News and Recent Outbreaks. 2020. Available from: https://www.newagebd.net/article/112198/ spread-of-dengue-feared-as-many-patients-go-unreported (accessed May 19, 2020).

[5] Chen N, Zhou M, Dong X, Qu J, Gong F, Han Y, et al. Epidemiological and clinical characteristics of 99 cases of 2019 novel coronavirus pneumonia in Wuhan, China: a descriptive study. Lancet 2020;395;507-13.

[6] Yan G, Pang L, Cook AR, Ho HJ, Win MS, Khoo AL, et al. Distinguishing Zika and dengue viruses through simple clinical assessment, Singapore. Emerg Infect Dis 2018;24;1565-8.

[7] DGHS. Guidelines, Directorate General of Health Services. 2020. Available from: https://dghs.gov.bd/index.php/bd/publication/ guideline (accessed May 19, 2020).

[8] The Daily Star. Double blow from deadly diseases | The Daily Star. 2020. Available from: https://www.thedailystar.net/ frontpage/news/double-blow-deadly-diseases-1902781 (accessed September 2, 2020).

[9] Joob B, Wiwanitkit V. COVID-19 can present with a rash and be mistaken for dengue. J Am Acad Dermatol 2020;82;e177. 
[10] Verduyn M, Allou N, Gazaille V, Andre M, Desroche T, Jaffar MC, et al. Co-infection of dengue and COVID-19: a case report. PLoS Negl Trop Dis 2020;14;e0008476.

[11] Saddique A, Rana MS, Alam MM, Ikram A, Usman M, Salman M, et al. Emergence of co-infection of COVID-19 and dengue: a serious public health threat. J Infect 2020 [Epub ahead of print].

[12] Kembuan GJ. Dengue serology in Indonesian COVID-19 patients: coinfection or serological overlap? IDCases 2020;22;e00927.
[13] Navarro JC, Arrivillaga-Henríquez J, Salazar-Loor J, RodriguezMorales AJ. COVID-19 and dengue, co-epidemics in Ecuador and other countries in Latin America: pushing strained health care systems over the edge. Travel Med Infect Dis 2020;37;101656.

[14] Harapan H, Ryan M, Yohan B, Abidin RS, Nainu F, Rakib A, et al. Covid-19 and dengue: double punches for dengue-endemic countries in Asia. Rev Med Virol 2020;e2161. 